\title{
Recent Progress in the Fundamental Understanding of Tropical Cyclone Motion
}

\author{
Kosuke ITO \\ Department of Physics and Earth Sciences, University of the Ryukyus, Nishihara, Japan \\ Chun-Chieh WU \\ Department of Atmospheric Sciences, National Taiwan University, Taiwan
}

Kelvin T. F. CHAN

School of Atmospheric Sciences, and Guangdong Province Key Laboratory for Climate Change

and Natural Disaster Studies, Sun Yat-sen University, China

Southern Marine Science and Engineering Guangdong Laboratory (Zhuhai), China

Ralf TOUMI

Department of Physics, Imperial College, UK

and

\section{Chris DAVIS}

Mesoscale and Microscale Meteorology Laboratory, National Center for Atmospheric Research, Colorado, USA

(Manuscript received 27 April 2019, in final form 22 June 2019)

\begin{abstract}
While the fundamental understanding of tropical cyclone (TC) movement is fairly mature, notable advancements are still being made. This paper summarizes new concepts and updates to the existing fundamental theories on TC movement obtained from simplified barotropic models, full-physics models, and data analysis, particularly since 2014. The scope includes recent works on the interaction between a TC and its environment, and the predictability related to TC movement. Although conventional concepts of steering flow, $\beta$-gyre, and diabatic heating remain important, a more complete understanding of TC movement governing mechanisms can provide an important basis for further track forecast improvements.
\end{abstract}

Keywords tropical cyclone motion; fundamental studies; multi-scale interactions

Citation Ito, K., C.-C. Wu, K. T. F. Chan, R. Toumi, and C. Davis, 2020: Recent progress in the fundamental understanding of tropical cyclone motion. J. Meteor. Soc. Japan, 98, 5-17, doi:10.2151/jmsj.2020-001.

Corresponding author: Kosuke Ito, Department of Physics and Earth Sciences, University of the Ryukyus, 1 Sembaru,

Nishihara, Okinawa 903-0213, Japan

E-mail: itokosk@sci.u-ryukyu.ac.jp

J-stage Advance Published Date: 8 October 2019

CThe Author(s) 2020. This is an open access article published by the Meteorological Society of Japan under a Creative Commons Attribution 4.0 International (CC BY 4.0) license (https://creativecommons.org/licenses/by/4.0). 


\section{Introduction}

Amongst all the elements of tropical cyclone (TC) forecasting, many forecasters consider the position (commonly referred to as "track") forecast to be the most important metric, because the forecasts of intensity, rainfall, and storm surge become less meaningful if the track forecast is incorrect. Tropical cyclone movement is largely controlled by the synoptic-scale flow but is also affected by the $\beta$-gyre effect and diabatic heating. Because of the importance of the TC track, extensive studies have been carried out on the effects of topography, land-sea contrast, and the impacts of interaction with other systems on TC motion.

Recently, however, considerable progress has been made in understanding the movement of TCs. With track forecast errors decreasing over several decades, consideration of the physical processes previously regarded as "minor" have become more important. Additionally, highlighting the updated understanding of well-known mechanisms and the fundamental aspect of predictability in regards to TC movement is important.

Therefore, this paper reviews the very recent fundamental findings that serve as an important basis for further track forecasting improvements. We do not repeat the detailed discussions on the concepts mentioned earlier in the excellent reviews by Elsberry (1995) and Chan $(2010,2017)$, nor those in the report on TC track in 8th International Workshop on Tropical Cyclones (IWTC-8) by Elliot and Yamaguchi (2014). In doing so, the focus is predominantly placed on fundamental and theoretical progress since 2014. Although recent advances in the forecasting skills of operational centers and new forecast techniques are outside the scope of this review, more details can be found in the review by Heming et al. (2019) of the recent progress in TC track forecasting and the expression of uncertainties, while Magnusson et al. (2019) reviewed recent advances in understanding difficult cases for track forecasts.

The paper is organized as follows: In Section 2, the conventional concept of steering is revisited. In Section 3, updated understandings obtained from a non-divergent barotropic model are introduced. Sections 4-7 describe the new findings and updates regarding recurvature, the influence of terrain, airsea interaction, and large-scale features, respectively. Section 8 outlines recent studies on large forecast error dynamics. In Section 9, our conclusions are summarized.

\section{Revisiting the concept of steering flow}

Previous studies agree that TC motion is primarily driven by the environmental steering flow (e.g., Chan and Gray 1982). Environmental steering wind is driven by synoptic-scale features in the vicinity of the TC and the definition can be broadened to include the effect of mesoscale features and subsynoptic-scale asymmetric circulations, such as $\beta$-gyres generated by the interaction between the TC circulation and the planetary vorticity gradient. An optimal environmental steering flow, obtained by varying the vertical extent of the steering layer and the radius, has successfully explained the TC track in many cases (e.g., Velden and Leslie 1991; Galarneau, Jr. and Davis 2013).

Recently, Wu and Chen (2016) conducted a potential vorticity tendency (PVT) diagnosis for the subkilometer high-resolution simulation of Typhoon Matsa (2005) to revisit the importance of steering flow in TC motion. They found that conventional deeplayer-mean steering, which is optimally determined within a certain radius from the TC center (defined as the geometric center of the circle on which the azimuthal-mean tangential wind reaches a maximum), plays a dominant role in TC motion because contributions from other processes are largely canceled out due to the coherent TC circulation structure. Conventional steering approximately accounted for the combined effect of the symmetric potential vorticity advection by the asymmetric flow and the contribution from the wavenumber 1 potential vorticity component advection by the symmetric flow, except short-time fluctuations (Fig. 1). Therefore, conventional steering represents TC motion by taking reasonable time-period averages.

They also argue that the short-time fluctuations cause the deviation of the conventional steering from the instantaneous TC motion. Trochoidal motion reasonably explained the observed phenomenon and was replicated in their high-resolution simulation as an oscillation of the TC motion speed with respect to the $9 \mathrm{~h}$ running mean. Although the displacement from the $9 \mathrm{~h}$ running mean track is usually less than $6 \mathrm{~km}$, conventional deep-layer-mean steering flow cannot account for such fluctuations.

\section{Barotropic framework}

Recently, Gonzalez III et al. (2015) have examined linear $\beta$-gyres as a stream function dipole with a uniform southeasterly ventilation flow across the vortex obtained from a barotropic non-divergent 

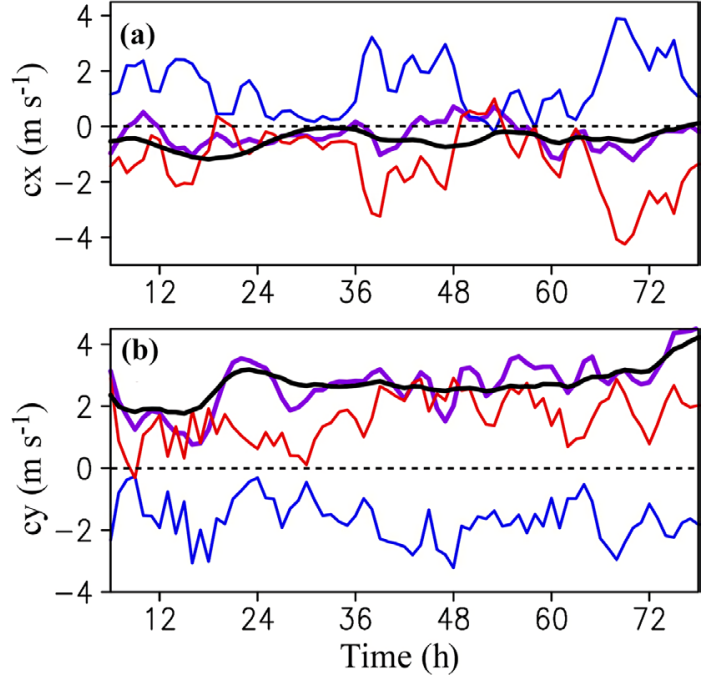

Fig. 1. Time series conventional steering data (thick black) and the contributions of the horizontal advection (HA) in the PVT equation (thick purple): (a) zonal component and (b) meridional component. The composition of HA includes the advection of the symmetric potential vorticity component by the asymmetric flow (HA1) and the advection of the wavenumber 1 potential vorticity component by the symmetric flow (HA2) terms. The anomaly in HA1 with respect to the conventional steering is shown in red, while that in HA2 is shown in blue (after $\mathrm{Wu}$ and Chen 2016).

model ${ }^{1}$. The $\beta$-gyre effect can be interpreted in terms of a wavenumber 1 vortex Rossby wave. According to Cotto et al. (2015), the frequencies of vortex Rossby waves in cylindrical coordinates are confined to a "passband" between zero frequency and the theoretical limit, illustrated in Fig. 2. Because the positive mean radial gradient of the vorticity in the outer region constitutes an outer waveguide that supports very-low-frequency vortex Rossby waves, the reproduced structure of the $\beta$-gyre clearly depends on the radial profile of the basic vorticity field. In other words, the TC translation speed associated with the $\beta$-gyre depends on the outer basic vorticity profile.

They also show that although the storm accelerated to unrealistically fast speed $\left(8.5 \mathrm{~m} \mathrm{~s}^{-1}\right)$ when simulated by a linear model, the introduction of non-linearity produces forced wavenumber 1 gyres that have the

\footnotetext{
${ }^{1}$ In this paper, primary circulation of a TC is anticlockwise unless otherwise noted.
}

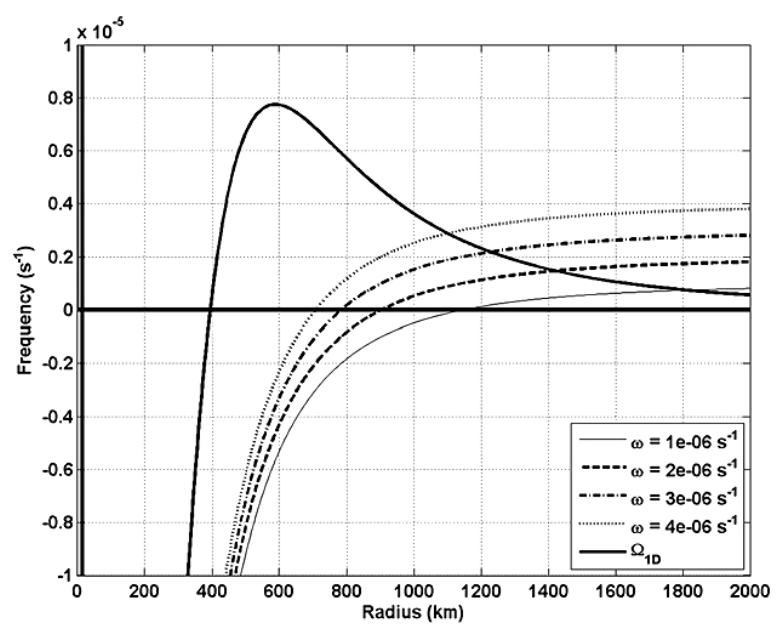

Fig. 2. Radial variation of Doppler-shifted frequency on the periphery of the vortex from Wood and White (2011) for different very low values of rotation frequency with respect to the ground, $\omega$. The outer waveguide lies between zero Dopplershifted frequency and $\Omega_{1 D}$, the frequency of a one-dimensional Rossby wave with the same tangential wavenumber and rotation frequency (after Gonzalez III et al. 2015). (C) American Meteorological Society. Used with permission.

opposite phase to that of the linear gyres. In their simulation, the ventilation flow counteracted the advection by the linear gyres, thus limiting the overall vortex speed up to approximately $3 \mathrm{~m} \mathrm{~s}^{-1}$.

Scheck et al. (2014) used a non-divergent barotropic model to investigate the structure and evolution of singular values (SVs) for a stable TC-like vortex in background flows with horizontal shear (Fig. 3). An analysis of anticyclonic shear with westerly wind to the north indicated that the leading initial SV for a stable TC-like vortex was aligned with streamlines connected to stagnation points. Additionally, the leading SV for anticyclonic shear was larger than that for the cyclonic shear. The evolved SV indicated a southwest-northeast displacement of the vortex. This was associated with the efficient advection by the outer perturbation. Their work suggests that decreasing errors directly related to stagnation points is important for improving track forecasts of TCs in anticyclonic shear with a westerly wind to the north.

\section{Recurvature}

Recurvature is usually explained as the change in environmental flow that steers the TC. Recent works have demonstrated that the recurvature occurrence and 

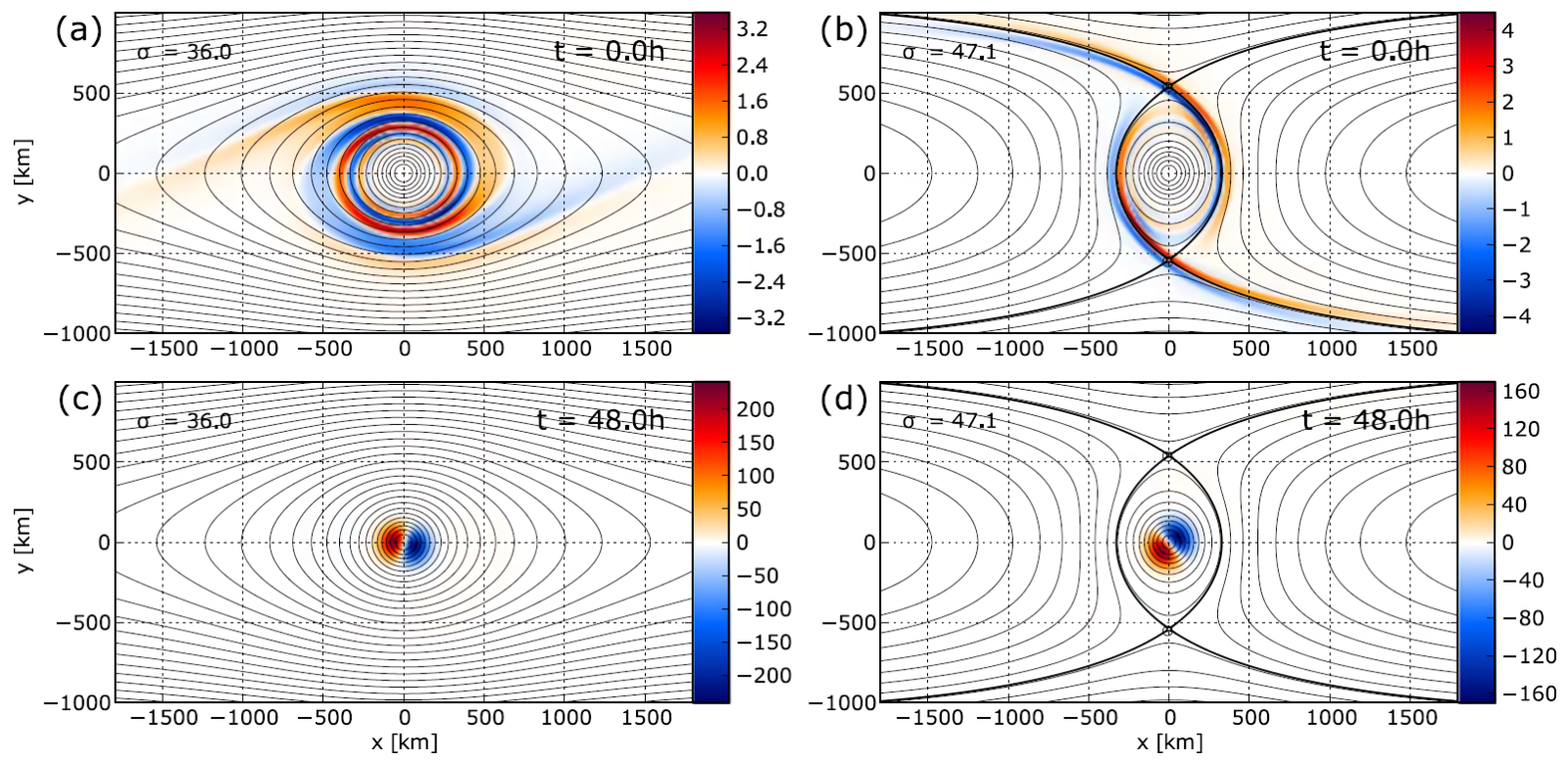

Fig. 3. Vorticity distribution of the initial SVs (a), (b) and the evolved SVs (c), (d) for a single stable vortex under an optimization time interval of 2 days and background cyclonic shears (left) and anticyclonic shears (right). The thin black contours are streamlines. In the anticyclonic shear case, the thick lines are the streamlines connected to the stagnation points (small circles) north and south of the vortex. The singular value, $\sigma$, is shown in the upper left of each panel (after Scheck et al. 2014).

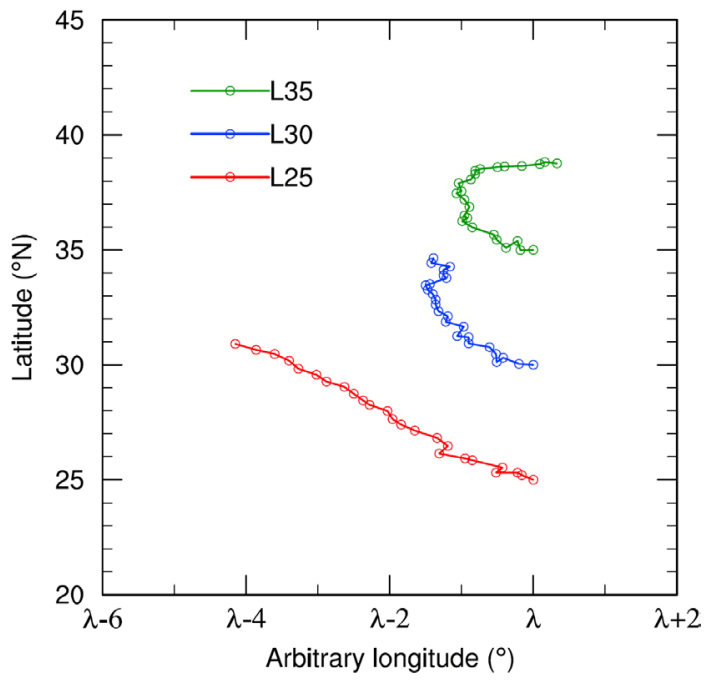

Fig. 4. Six-day TC tracks for a symmetric spun-up vortex initially at $25^{\circ} \mathrm{N}, 30^{\circ} \mathrm{N}$, and $35^{\circ} \mathrm{N}$ with no background flow. The time interval between each track marker is 6 hours. The tracks are drawn based on the central minimum sea level pressure of the vortex (after Chan and Chan 2016). its location are sensitive to the sea surface temperature (SST) field and the TC size, as discussed in Sections 5 and 6 . Notably, it has been found that a TC located at a higher latitude can recurve by itself, even in the absence of a background flow (Chan and Chan 2016; Fig. 4). Differential horizontal advection of the planetary vorticity due to TC circulation at different vertical levels causes vertical wind shear (known as $\beta$-shear), upper tropospheric anticyclones, and asymmetric distributions of convection to develop. The upper tropospheric anticyclone flow on the equatorward side of the TC and the diabatic heating associated with asymmetric convection combine to cause the TC to recurve intrinsically. Given that a TC located at a higher latitude develops more slowly (Li et al. 2012), the anticyclonic outflow of a higher-latitude TC advects the upper tropospheric anticyclone less outwardly (see Fig. 3 of Chan and Chan (2016)). As a result, the upper tropospheric anticyclone of a higher-latitude TC is located closer to the vortex, and thus influences TC movement more substantially. Subsequently, the convection and diabatic heating asymmetries cause the intrinsic recurvature at higher latitudes. Particularly when environmental steering flow is weak, this mechanism is thought to contribute to the movement of TCs at a higher latitude. 


\section{Influence of topography}

The interaction between the terrain, basic flow, and TC is non-linear. Recent studies have made efforts to further understand the mechanisms that induce TC track deflection (Lin et al. 2016). The following mechanisms have been proposed to explain upstream track deflection: (1) advection by orographically blocking basic flow, (2) channeling effects, (3) asymmetric latent heating, (4) asymmetric middle-level steering flow, (5) terrain-induced gyres, and (6) approach angles and landing location.

Tang and Chan (2014) analyzed the effect of terrain in Taiwan and the Philippines. Terrain-induced gyres were found over both the Central Mountain Range of Taiwan and the mountains of Luzon. A pair of terrain-induced gyres rotated cyclonically around the $\mathrm{TC}$, and the associated flow began to push the TC northward (Fig. 5). As the TC neared landfall, the anticyclonic gyre was located north of the TC in contrast to southern position of the cyclonic gyre. Furthermore, the height of the terrain was confirmed to control the strength of the terrain-induced gyre, with a weaker gyre produced in the Philippines terrain simulation. The PVT diagnosis revealed that while horizontal advection played a major role, diabatic heating became comparably important during the landfall period.

Jian and $\mathrm{Wu}$ (2008) and Huang et al. (2011) suggested that the low-level channeling effect is the primary contributor to track deflection for a TC that is very close to land. Specifically, a low-level northerly jet can form in the western quadrant channel of an approaching westward-moving storm in which the presence of terrain constrains the inner-core circulation. $\mathrm{Wu}$ et al. (2015) investigated a different mechanism related to the sudden track changes observed in TCs approaching Taiwan. In one key finding of the study, the azimuthally asymmetric tangential wind at middle levels was identified as the robust flow characteristic during the southward turn of a TC, instead of that at low levels. The azimuthal change in the wind speed is connected to the azimuthal change in vertical velocity. Sensitivity experiments for different parameters demonstrated that southward track deflection is a common phenomenon prior to landfall. The results also suggested that in regions with higher terrain, a TC approaches the northern part of the terrain, while at reduced translation speeds, TC track deflection would be prominent. Other parameters, such as the mountain width and length, and the radius of maximum wind, exhibited relatively limited effects on the TC track.

Lin et al. (2016) used the vorticity tendency (VT)

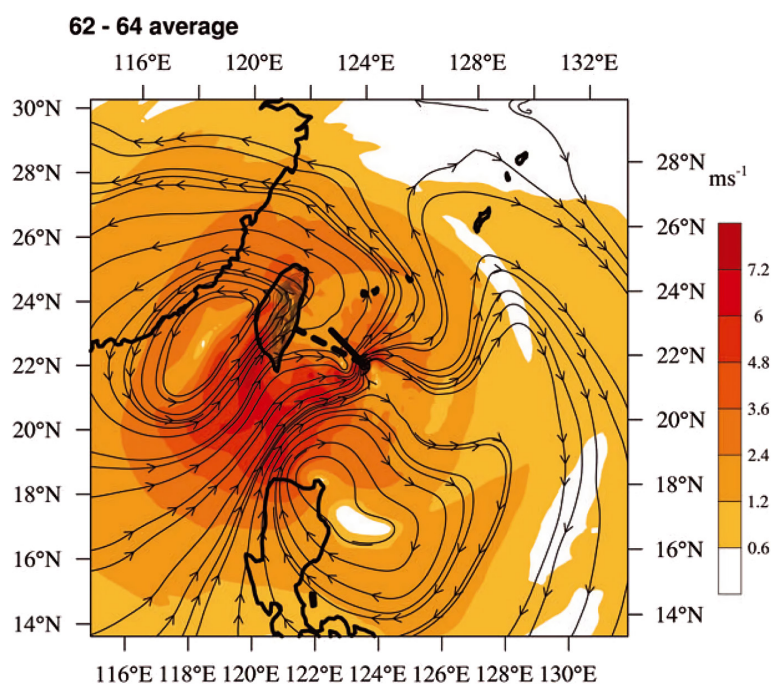

Fig. 5. Three-hour (62-64 h) averaged asymmetric flow differences between the landfall and control cases within a $0.860-0.620$ eta level range. Thin lines with arrows represent the streamlines of the difference between the cases, while shadings show the wind-speed difference in $\mathrm{m} \mathrm{s}^{-1}$. The solid straight line represents the overall direction of the TC in the case with Taiwan, and the dotted line that of the case without Taiwan (adopted from and modified after Tang and Chan 2014).

diagnostic to examine the southward deflection of a TC. When the TC was upstream of the mountain in the study, orographic blocking caused the easterly basic flow to become sub-geostrophic. The TC decelerated and was deflected to the south, with the VT primarily dominated by horizontal advection (see Fig. 1 of Lin et al. (2016)). Subsequently, the TC cleared the mountain clockwise, steered by the orographically generated high-pressure. During this time, the VT was mainly comprised of the horizontal advection and stretching terms. Moreover, diabatic heating was analyzed in connection to the northwestward movement over the leeward slope. The enhanced advection of eyewall convective clouds associated with diabatic heating contributed to the abrupt northwesterly turn of the TC.

Both the channeling effect and asymmetries in the mid-levels contribute to the southward track deflection of a TC. Huang and Wu (2018) investigated the southward deflection of a westward-moving TC oriented toward idealized terrain, similar to that in Taiwan. Analyses of both the flow asymmetries and PVT demonstrated that horizontal advection contributed to the southward movement of the TC (see Fig. 9 
of Huang and $\mathrm{Wu}$ (2018)). The track deflection was examined over two separate time periods, in which different mechanisms lead to the southward movement. The terrain-induced changes in the background flow initially caused the large-scale steering current to push the TC southward, though the TC was still far from the terrain. As the TC approached the idealized topography, the inner-core dynamics became important. Figure 6 shows that the TC-terrain-induced chan-

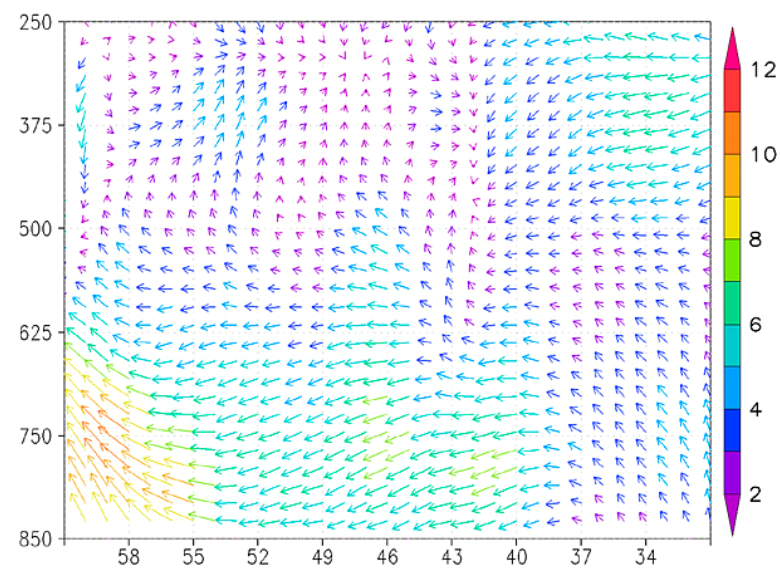

Fig. 6. Asymmetric horizontal flow $\left(\mathrm{m} \mathrm{s}^{-1}\right)$ at different levels and times. Each vector indicates the zonal and meridional components calculated within $100 \mathrm{~km}$ of the vortex. The $\mathrm{x}$-axis shows the integration time (h), and y-axis the vertical levels in terms of atmospheric pressure $(\mathrm{hPa})$ (after Huang and Wu 2018). neling effect resulted in further southward deflection of the track during the integration time, $t$, of $40-50$ h. During this period, asymmetries also developed in the mid-level flow (Fig. 6), in part associated with the vertical momentum transport effect. The combination of a large-scale environmental flow, low-level channeling effect, and asymmetries in mid-level flow all contribute to southward TC track deflection.

\section{Role of atmosphere-ocean interaction}

Recent research suggests that the initial SST can also affect TC tracks. Katsube and Inatsu (2016) and Sun et al. (2017) have found that warmer SSTs stimulate earlier northward TC recurvature in some cases in the western North Pacific (WNP). Sun et al. (2017) ascribed this result to the retreat of the subtropical high in the higher SST experiment (Fig. 7). Based on analysis using a simplified linear baroclinic model, Katsube and Inatsu (2016) interpreted this track change as the well-known subtropical thermal response documented by Hoskins and Karoly (1981) (Fig. 8).

While persistent SST forcing can affect TC tracks, the direct impact of storm-induced oceanic feedback on the TC track is less obvious. Modeling studies have not revealed strong impacts of atmosphere-ocean coupling on TC tracks. The range of investigations conducted includes idealized TC studies with and without ocean coupling (e.g., Zhu et al. 2004; Duan et al. 2013), a study of the impacts of adding wave coupling (Liu et al. 2011), and individual case studies in the Atlantic (e.g., Winterbottom et al. 2012). To obtain (a) Simulations in Songda (2004) case

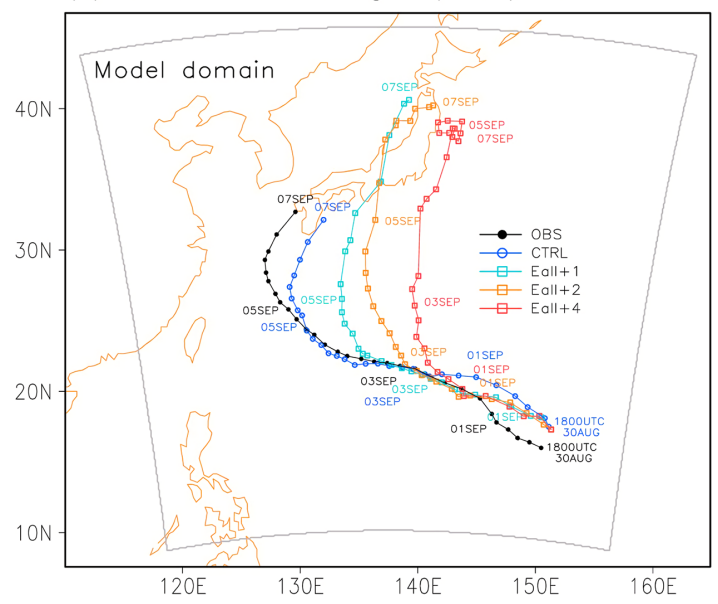

(b) Simulations in Megi (2010) case

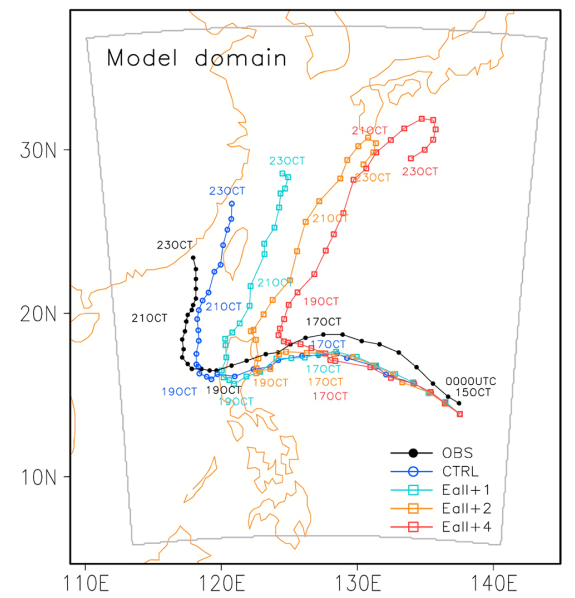

Fig. 7. Storm tracks at $6 \mathrm{~h}$ intervals simulated in sensitivity experiments with various underlying SSTs, and the observed best TC track for (a) Songda (2004) and (b) Megi (2010) (after Sun et al. 2017). 


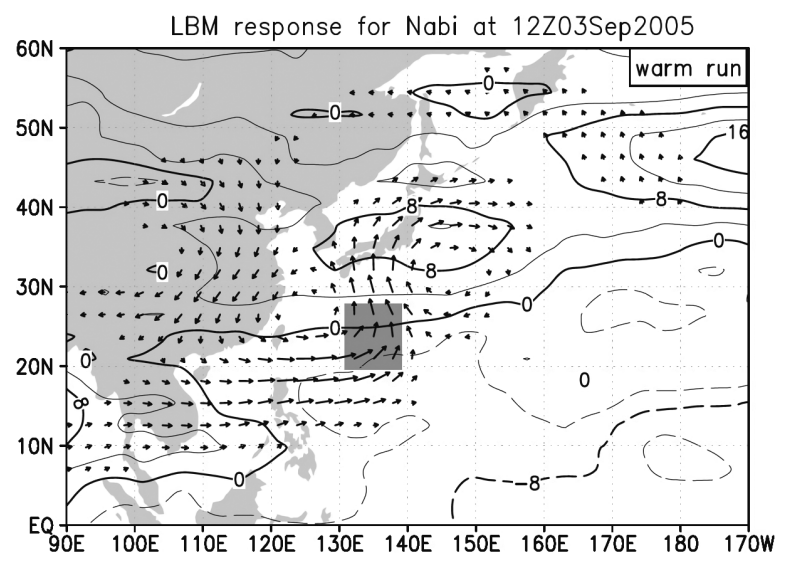

Fig. 8. Steering flow, as calculated from the stationary linear response to diabatic heating, at the cyclone center at 1200 UTC 3 Sep 2005 obtained from the warm run of the Nabi experiment. A basic-state zonal wind at $500 \mathrm{hPa}$ is superimposed ( $\mathrm{m} \mathrm{s}^{-1}$; contours at a $4 \mathrm{~m} \mathrm{~s}^{-1}$ interval, with negative contours dashed). Diabatic heating is imposed in the shaded region (adopted and modified after Katsube and Inatsu 2016). (C) American Meteorological Society. Used with permission.

more reliable results, Ito et al. (2015) analyzed 34 TC cases (281 simulations) near Japan and found that the atmosphere-ocean coupling had a minor effect on the TC track. They reported that the atmosphere-ocean coupling slightly deflected the TC position to the left (by roughly $20 \mathrm{~km}$ ) during the forecast time of 36 hours. Presumably, this effect occurs because significant sea surface cooling on the right-hand side is not favorable to convective activities, leading to the genesis of potential vorticity (Fig. 9).

However, secondary and indirect influences of ocean coupling on the track due to changes in intensity may occur in certain cases. In the case of TC Fanapi (2010), storm-induced cooling weakened the vertical coherence of the TC. A weakening, shallower cyclone may be more sensitive to lower level flow. Thus, as coupling reduces the intensity, the effective steering flow may change (Lin et al. 2018). Coupling with the ocean could indirectly change the upper-level anticyclone of the TC through changes in intensity (diabatic heating) and the convection symmetry. Therefore, the track may respond to the flow differently. Srinivas et al. (2016) used a coupled model and an AMSR-E-based initial condition for six TCs in the Bay of Bengal and yielded a $14 \%$ improvement in the 3-day track forecast over the fixed National Oceanic and Atmospheric

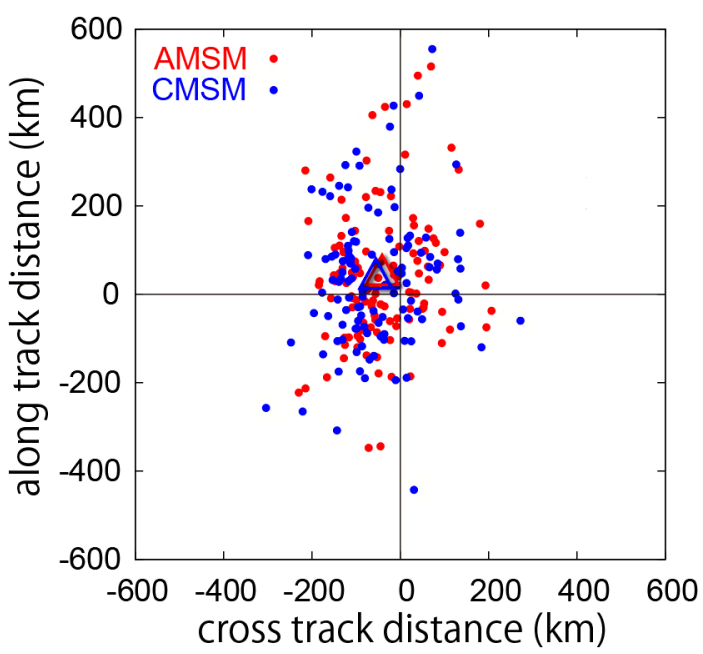

Fig. 9. Tropical cyclone center position at $\mathrm{T}+36$ $\mathrm{h}$ for each experiment with the non-hydrostatic atmospheric (red) and coupled (blue) models relative to the best track from the Regional Specialized Meteorological Center Tokyo. The vertical axis is the along-track distance in the direction of the modeled TC motion from $\mathrm{T}+30$ to $\mathrm{T}+36 \mathrm{~h}$, while the horizontal axis is the cross-track distance. Triangles in the same color indicate the mean positional bias (adopted and modified after Ito et al. 2015).

\section{Administration SST.}

It is important to distinguish between the role of storm-induced cooling on a short-term time scale, and seasonal or climate time scales. Ocean-coupling can alter large-scale SST patterns and consequently steering flow winds on longer time scales (e.g., Ogata et al. 2016; Sun et al. 2017). The seasonal forecast and climate projections of track densities are expected to be sensitive to atmosphere-ocean coupling. However, a recent climatological study of the entire Indian Ocean found that employing a coupled model had no significant impact on track density (Lengaigne et al. 2019).

\section{Large-scale features}

Sudden poleward track changes often occur when a TC is positioned east of a monsoon gyre (MG). An MG is defined as a low-frequency cyclonic (or anticyclonic) circulation in the lower (or upper) troposphere with a diameter of about 2000-2500 km (e.g., Lander 1994). Previous model studies have shown a binary interaction between an MG and a TC, in which the TC was advected cyclonically around and moved into the 


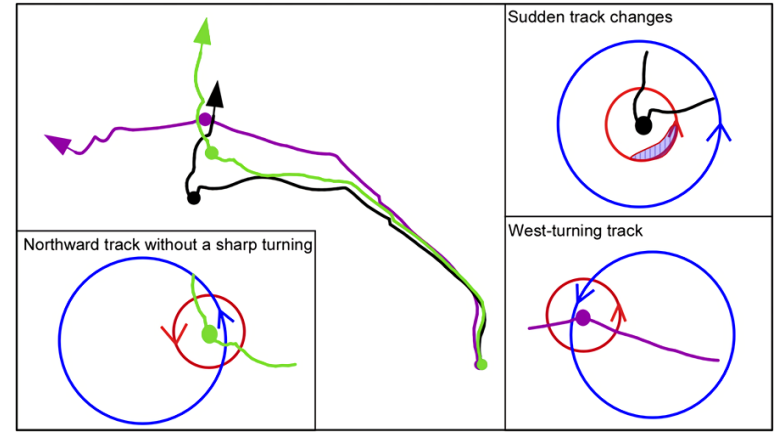

Fig. 10. Schematic diagrams of the three track types with an MG. The blue and red circles with arrows denote the MG and TC circulation, respectively (after Liang and Wu 2015). (C) American Meteorological Society. Used with permission.

center of the MG. Thereafter, a $\beta$-induced dispersion in the southeast produced northward steering (Carr III and Elsberry 1995). Recently, Liang and Wu (2015) investigated three types of tracks (sudden northward, northward without a sharp turn, and westward) that can occur when a TC is initially located in the eastern semicircle of an MG (Fig. 10). The investigated TCs that took westward tracks, and northward tracks without a sharp turn did not experience the coalescence process undergone in northward tracks with a sharp turn. Westward-turning TCs moved faster than MGs, while northward-turning TCs without a sharp turn moved more slowly than MGs. Additionally, they demonstrated that track-type is also sensitive to the initial position and the structure of the TCs and MGs. Ge et al. (2018) indicated that a sharp northward turn can also be influenced by the vertical structure and the intensity of an MG. In their idealized simulation, which considered a deeper and stronger MG, the total VT of the wavenumber 1 component of the TC nearly disappeared due to the vorticity advection of the MG. This means that the westward steering flow vanished when the centers of the TC and MG were almost collocated. After the TC decreased in speed, the TC exhibited a sharp northward turn due to the $\beta$-effect, consistent with a barotropic model simulation (Fig. 11). In contrast, a TC interacting with a shallower MG experienced a nearly constant northwestward track. The study additionally showed that the differences in the radial gradient of the relative vorticity also yielded similar track differences in a simple barotropic model. Bi et al. (2015) showed that the sharp northward turn of Typhoon Megi (2010) was induced by the strength

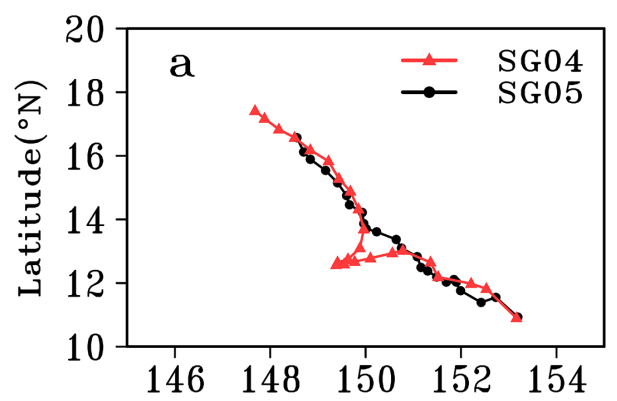

Fig. 11. Simulated 3-hourly TC tracks with a deep intense MG (SG04) and a shallow weak MG (SG05) (after Ge et al. 2018). (C) American Meteorological Society. Used with permission.

of the TC in the initial field, as no sharp turn was observed when the initial TC was weakened. These works suggest that the TC track depends on both the low-frequency flows associated with the MG as well as the TC vortex itself.

Wei et al. (2016) statistically investigated the relationship between the upper tropospheric cold low (UTCL) and the TC tracks over the WNP during 20002012. They found that for all the TCs and UCTLs within a 15-degree interaction distance, the UTCL had little impact on the average directional change of the TC track, contrary to the results obtained by previous case studies. Albeit with the lower frequency, most of the left-turning TCs within a 5-degree distance experienced abrupt left-turning, up to 50 degrees in 12 hours (Fig. 12). The TCs also tended to slow down during abrupt directional changes.

Sun et al. (2015) investigated the interaction between TCs and subtropical highs over the WNP, focusing on the initial size of the TC in the TC Songda (2004) and Megi (2010) cases. With an increase in the initial storm size, the main body of a subtropical high tended to withdraw, and TCs initially located on the southwestern edge of the subtropical high tended to turn northwards earlier (Fig. 13). The increase in mass flux due to the larger vortex decreased the middle troposphere geopotential height in the outer region of the TC, thus leading to a break of the subtropical high in the WNP.

\section{Dynamics of large forecast errors in TC tracks}

Track forecast errors have generally decreased, owing to increased observations and substantial improvements in numerical weather prediction systems. Recently, Landsea and Cangialosi (2018) posted an interesting discussion topic on the limits that have been 


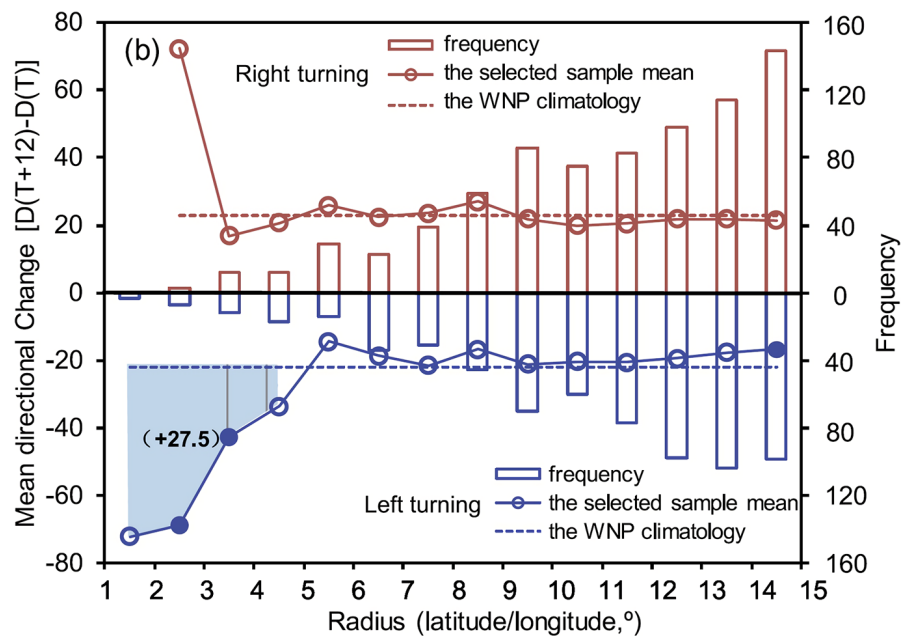

Fig. 12. The frequency (columns) and mean directional change (lines with small circles; unit: 8) of TCs in $12 \mathrm{~h}$, as a function of the distance from the composite UTCL center for the selected right-turning (brown) and left turning (blue) cases. The distance is measured in degrees by $r=\sqrt{(\Delta \mathrm{lon})^{2}+(\Delta \mathrm{lat})^{2}}$, where $\Delta$ lon and $\Delta$ lat are the differences in latitude and longitude of the two locations, respectively. Filled circles show significant differences at a $95 \%$ confidence level from the corresponding WNP climatology, as indicated by dashed lines. The quantity in parentheses shows a significant increase in the mean directional change of the TCs in the shaded distance range throughout the corresponding WNP climatology (after Wei et al. 2016). (C) American Meteorological Society. Used with permission.
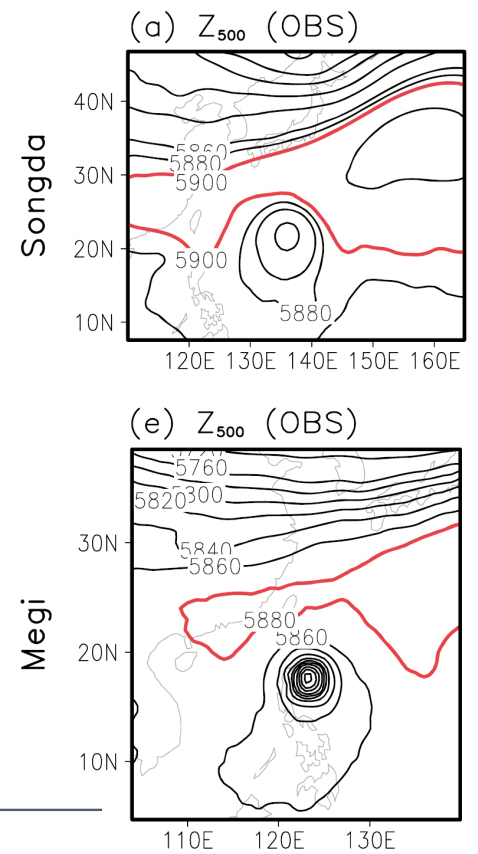

(b) $Z_{500}$ (ES)

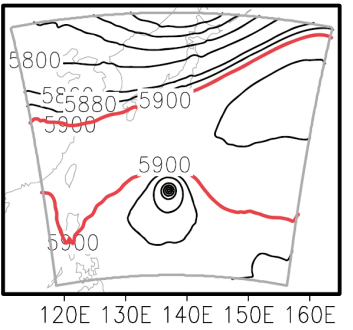

(f) $Z_{500}$ (ES)

(c) $Z_{500}(E M)$
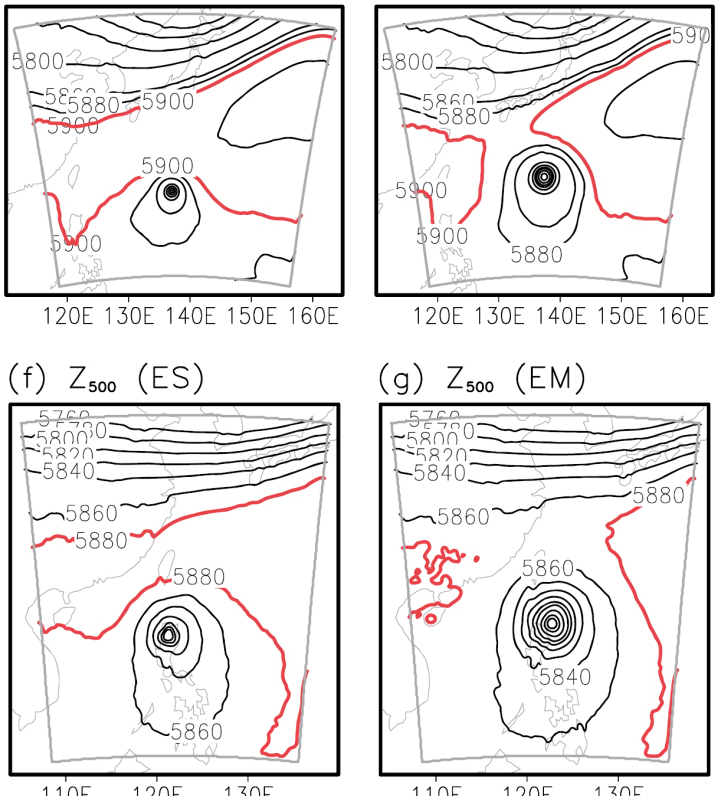

(g) $Z_{500}$ (EM)

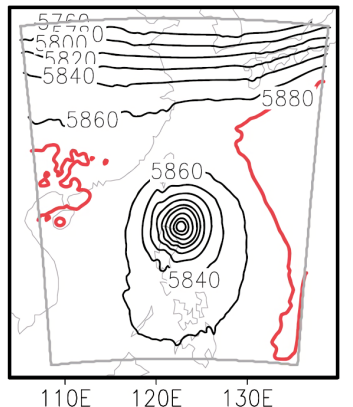

(d) $Z_{500}(E L)$

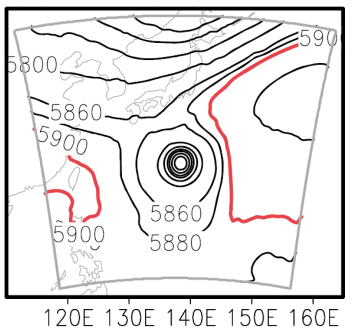

(h) $Z_{500}(E L)$

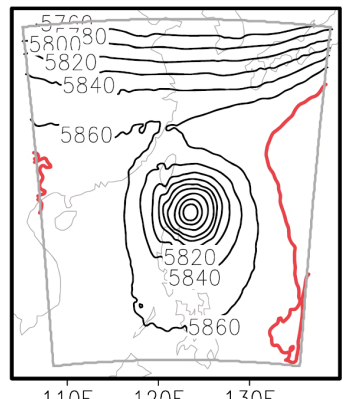

Fig. 13. The geopotential height at $500 \mathrm{hPa}$ from NCEP reanalysis data and from simulations at 0000 UTC 3 September 2004 and 0000 UTC 18 October 2010, corresponding to the cases of $(a-c)$ Songda (2004) and (d-f) Megi (2010), respectively. The contour of $5900 \mathrm{~m}$ in the Songda case and the contour of $5880 \mathrm{~m}$ in the Megi case are highlighted in red (after Sun et al. 2015). 
reached in TC track predictability. However, there are still forecast busts, in which track forecast errors are very large (Yamaguchi et al. 2017; Magnusson et al. 2019). The investigation of such cases remains one of the major issues for TC researchers and operational centers.

The theoretical reasons for large track errors were explored by Torn et al. (2018) in the cases of Debby (2012), Joaquin (2015), and Lionrock (2016). Using ensemble sensitivity analysis, the authors concluded that the foremost factor in creating a large ensemble spread was a steering flow pattern with a saddle point of strong deformation (Fig. 14) downstream of the immediate TC location. In this pattern, small perturbations to the initial state had drastic consequences for track prediction 2-4 days later.

Yamada et al. (2016) investigated the large northward bias seen in the Japan Meteorological Agency Global Spectral Model (JMA-GSM) for TC Fensheng (2008), in which the track was successfully simulated using a Nonhydrostatic Icosahedral Atmospheric Model (NICAM) with finer grid spacing. Their diagnosis using vorticity budget attributed the large bias to the marked asymmetry of the rainfall, concentrated in the downshear side (Fig. 15). Although both models could reproduce the asymmetric structure due to the vertical wind shear, they exhibited large differences in the vertical structure and the magnitude of the stretching term. The results suggest that storm-scale heating profile usage as a response to persistent vertical shear can induce large track forecast errors in some cases.

Saunders et al. (2019) demonstrated that the notable hurricane track of hurricane Joaquin (2015) had significant sensitivity to the choice of cumulus convection. Their diagnosis suggested that the hurricane core region structure, including the vertical extent of the diabatic heating, vertical velocity, and relative humidity, could also contribute substantially to track errors, along with changes in large-scale flow. Specifically, the asymmetry and local absolute vorticity tendency throughout the inner-core region and in its vicinity had a strong effect on Joaquin's hairpin turn.

\section{Concluding remarks}

Highly idealized models provide an important perspective for the forecasting of a TC track. Recent works suggest that TC track forecasting is sensitive to the relative vorticity radial profile in the outer region of the TC, as this profile is related to the $\beta$-gyre and the faster development mode. Recent idealized simulations using full-physics models have provided deeper understandings of existing mechanisms, as

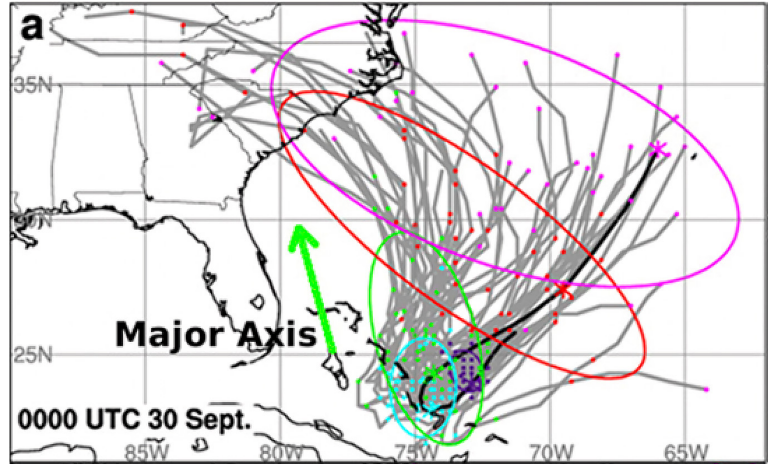

Fig. 14. European Centre for Medium-Range Weather Forecasts ensemble forecasts of hurricane Joaquin initialized at 0000 UTC 30 September 2015. The dots indicate the location of each ensemble member at 24-h intervals, while the colored circles show a bivariate normal fit for the positions in each interval. Purple denotes 24-h locations, cyan denotes 48-h locations, green denotes 72-h locations, red denotes 96-h locations, and magenta denotes $120-\mathrm{h}$ locations. The thick black line denotes the National Hurricane Center best track positions, while the stars indicate the corresponding best track position each 24 -h interval. The direction of the 48-h major axis is denoted by the green vector (after Torn et al. 2018).

well as some previously unknown mechanisms: the steering flow concept, the intrinsic recurving nature, the channeling effect in the middle troposphere, the atmosphere-ocean interaction, and the impact of largescale features. It has been suggested that the horizontal and vertical structure of a TC affects its own track either independently or through interactions with other systems. State-of-the-art models have demonstrated the ability to represent some of these effects to a certain extent. These findings are furthermore important for understanding the mechanisms governing TC track changes, particularly in cases where the TC tracks were changed by using finer meshes, new physical schemes, or based on the topography and coastline. More accurate future implementation of these components is highly desirable.

The fundamental aspects of TC movement are also important because they can facilitate observation and data assimilation system design. Recent works suggest that for TCs in a synoptic-scale anticyclonic shear, uncertainties along streamlines connected to stagnation points should be reduced. The recent case studies suggest that large track forecast errors can result from 

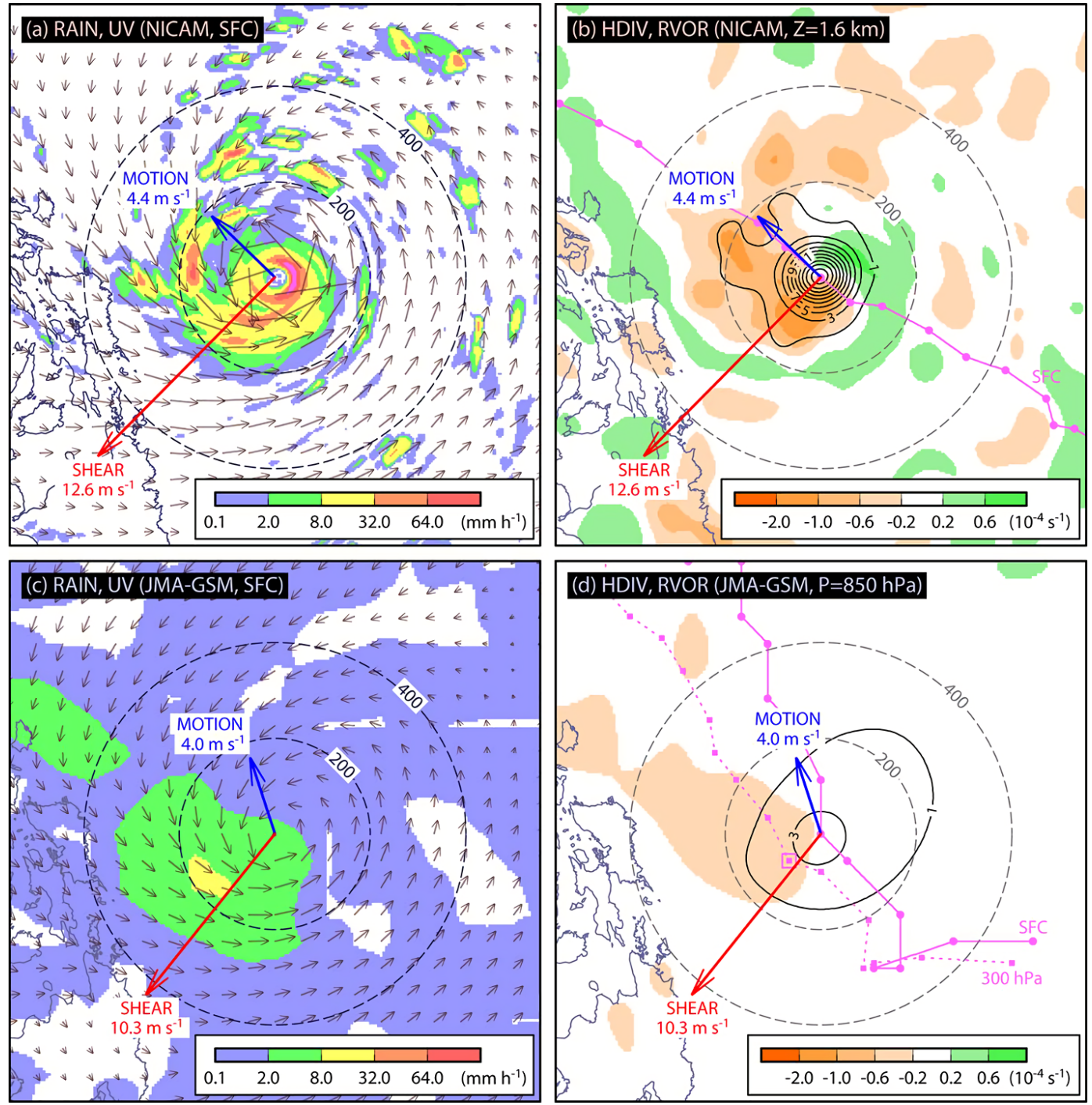

Fig. 15. Horizontal distributions at 0000 UTC 20 June simulated by NICAM (top) and JMA-GSM (bottom). The left panels show the rain rate (shading) and surface wind vectors. The right panels show the horizontal divergence (shading) and the relative vorticity (contour) at $1.6 \mathrm{~km} \mathrm{MSL}$ or $850 \mathrm{hPa}$. Broken circles indicate 200- and 400-km ranges from the surface vortex center. Blue and red arrows indicate the TC motion and vertical shear, respectively. The 6-hourly positions of the center at the surface and at $300 \mathrm{hPa}$ are shown by circles with solid lines and rectangles with dotted lines, respectively (after Yamada et al. 2016).

both the strong deformation intrinsically produced in a large-scale flow pattern with a saddle point, as well as failures to reproduce the storm-scale asymmetric heating profile.

As a result of sophisticated numerical modeling and observations, TC position forecast errors have decreased to less than $100 \mathrm{~km}$ at 24 hours and 200-300 $\mathrm{km}$ at 72 hours. Consequently, the physical processes previously considered "minor" have become "substan- tial" in importance, as the required accuracy level has become higher. In addition, further understanding of large forecast errors and unknown processes is needed. Although the conventional concepts of steering flow, $\beta$-gyre, and diabatic heating remain important, a better understanding of various physical processes should be regarded as an important step for disentangling the complicated dynamics associated with actual tropical cyclone movement. 


\section{Acknowledgments}

This paper was based on the subtopic report "Recent Progress in Fundamental and Theoretical Studies on TC motion" for 9th International Workshop on Tropical Cyclones (IWTC-9) held on 3-7 December 2018 in Honolulu, Hawaii, USA. This work of Kosuke Ito was supported by MEXT KAKENHI Grant 18H01283 and University of the Ryukyus Research Project Promotion Grant (Strategic Research Grant 18SP01302). The work of Chun-Chieh $\mathrm{Wu}$ is supported by the Ministry of Science and Technology of Taiwan under Grants MOST 106-2111-M-002-013-MY3. The work of Kelvin T. F. Chan was jointly supported by the National Natural Science Foundation of China (41775097 and 41975052), and the National Natural Science Foundation of China and Macau Science and Technology Development Joint Fund (NSFC-FDCT), China and Macau (41861164027). The contribution of Chris Davis is based upon work supported by the National Center for Atmospheric Research, which is a major facility sponsored by the National Science Foundation under Cooperative Agreement 1852977.

\section{References}

Bi, M., T. Li, M. Peng, and X. Shen, 2015: Interactions between Typhoon Megi (2010) and a low-frequency monsoon gyre. J. Atmos. Sci., 72, 2682-2702.

Carr III, L. E., and R. L. Elsberry, 1995: Monsoonal interactions leading to sudden tropical cyclone track changes. Mon. Wea. Rev., 123, 265-289.

Chan, J. C. L., 2010: Movement of tropical cyclone. Global Perspectives on Tropical Cyclones. World Scientific, 133-148.

Chan, J. C. L., 2017: Physical mechanisms responsible for track changes and rainfall distributions associated with tropical cyclone landfall. Oxford Handbooks Online, doi:10.1093/oxfordhb/9780190699420.013.16.

Chan, J. C. L., and W. M. Gray, 1982: Tropical cyclone movement and surrounding flow relationships. Mon. Wea. Rev., 110, 1354-1374.

Chan, K. T. F., and J. C. L. Chan, 2016: Tropical cyclone recurvature: An intrinsic property? Geophys. Res. Lett., 43, 8769-8774.

Cotto, A., I. Gonzalez III, and H. E. Willoughby, 2015: Synthesis of vortex Rossby waves. Part I: Episodically forced waves in the inner waveguide. J. Atmos. Sci., 72, 3940-3957.

Duan, Y., R. Wu, R. Yu, and X. Liang, 2013: Numerical simulation of changes in tropical cyclone intensity using a coupled air-sea model. Acta Meteor. Sin., 27, 658-672

Elliott, G., and M. Yamaguchi, 2014: Motion - recent advances. Proceeding of 7th International Workshop on
Tropical Cyclones, 44 pp. [Available at http://www. wmo.int/pages/prog/arep/wwrp/new/documents/ Topic1_AdvancesinForecastingMotion.pdf.]

Elsberry, R. L., 1995: Global Perspectives on Tropical Cyclones. WMO-/TD-No. 693, World Meteorological Organization, $289 \mathrm{pp}$.

Galarneau, T. J., Jr., and C. A. Davis, 2013: Diagnosing forecast errors in tropical cyclone motion. Mon. Wea. Rev., 141, 405-430.

Ge, X., Z. Yan, M. Peng, M. Bi, and T. Li, 2018: Sensitivity of tropical cyclone track to the vertical structure of a nearby monsoon gyre. J. Atmos. Sci., 75, 2017-2028.

Gonzales III, I., A. Cotto, and H. E. Willoughby, 2015: Synthesis of vortex Rossby waves. Part II: Vortex motion and waves in the outer waveguide. J. Atmos. Sci., 72, 3958-3974.

Heming, J. T., F. Prates, M. A. Bender, R. Bowyer, J. Cangialosi, P. Caroff, T. Coleman, J. D. Doyle, A. Dube, G. Faure, J. Fraser, B. C. Howell, Y. Igarashi, R. McTaggart-Cowan, M. Mohapatra, J. R. Moskaitis, J. Murtha, R. Rivett, M. Sharma, C. J. Short, A. A. Singh, V. Tallapragada, H. A. Titley, and Y. Xiao, 2019: Review of Recent Progress in Tropical Cyclone Track Forecasting and Expression of Uncertainties. Trop. Cyclone Res. Rev., 8, 181-218.

Hoskins, B. J., and D. J. Karoly, 1981: The steady linear response to a spherical atmosphere to thermal and orographic forcing. J. Atmos. Sci., 38, 1179-1196.

Huang, K.-C., and C.-C. Wu, 2018: The impact of idealized terrain on upstream tropical cyclone track. J. Atmos. Sci. 75, 3887-3910.

Huang, Y.-H., C.-C. Wu, and Y. Wang, 2011: The influence of island topography on typhoon track deflection. Mon. Wea. Rev., 139, 1708-1727.

Ito, K., T. Kuroda, K. Saito, and A. Wada, 2015: Forecasting a large number of tropical cyclone intensities around Japan using a high-resolution atmosphere-ocean coupled model. Wea. Forecasting, 30, 793-808.

Jian, G.-J., and C.-C.Wu, 2008: A numerical study of the track deflection of Supertyphoon Haitang (2005) prior to its landfall in Taiwan. Mon. Wea. Rev., 136, 598-615.

Katsube, K., and M. Inatsu, 2016: Response of tropical cyclone tracks to sea surface temperature in the western North Pacific. J. Climate, 29, 1955-1975.

Lander, M. A., 1994: Description of a monsoon gyre and its effects on the tropical cyclones in the western North Pacific during August 1991. Wea. Forecasting, 9, 640-654.

Landsea, C. W., and J. P. Cangialosi, 2018: Have we Reached the limits of predictability for tropical cyclone track forecasting? Bull. Amer. Meteor. Soc., 99, 2237-2243.

Lengaigne, M., S. Neetu, G. Samson, J. Vialard, K. S. Krishnamohan, S. Masson, S. Jullien, I. Suresh, and C. E. Menkes, 2019: Influence of air-sea coupling on Indian 
Ocean tropical cyclones. Climate Dyn., 52, 577-598.

Li, T., X. Ge, M. Peng, and W. Wang, 2012: Dependence of tropical cyclone intensification on the Coriolis parameter. Trop. Cyclone Res. Rev., 1, 242-253.

Liang, J., and L. Wu, 2015: Sudden track changes of tropical cyclones in monsoon gyres: Full-physics, idealized numerical experiments. J. Atmos. Sci., 72, 1307-1322.

Lin, K.-J., S. S. Chen, and S.-C. Yang, 2018: Ensemble forecast of typhoon Fanapi (2010) using a high-resolution coupled model: Effects of TC-ocean interaction. Proceeding of 33rd conference on hurricanes and tropical meteorology. Amer. Meteor. Soc., 3 pp. [Available at https://ams.confex.com/ams/33HURRICANE/web program/Paper340525.html.]

Lin, Y.-L., S.-H. Chen, and L. Liu, 2016: Orographic influence on basic flow and cyclone circulation and their impacts on track deflection of an idealized tropical cyclone. J. Atmos. Sci., 73, 3951-3974.

Liu, B., H. Liu, L. Xie, C. Guan, and D. Zhao, 2011: A coupled atmosphere-wave-ocean modeling system: Simulation of the intensity of an idealized tropical cyclone. Mon. Wea. Rev., 139, 132-152.

Magnusson, L., J. D. Doyle, W. A. Komaromi, R. D. Torn, C. K. Tang, J. C. L. Chan, M. Yamaguchi, and F. Zhang, 2019: Advances in understanding difficult cases of tropical cyclone track forecasts. Trop. Cyclone Res. Rev., 8, 109-122.

Ogata, T., R. Mizuta, Y. Adachi, H. Murakami, and T. Ose, 2016: Atmosphere-ocean coupling effect on intense tropical cyclone distribution and its future change with 60 km-AOGCM. Sci. Rep., 6, 29800, doi:10.1038/srep 29800.

Saunders, P., Y. Yu, and Z. Pu, 2019: Sensitivity of numerical simulations of hurricane Joaquin (2015) to cumulus parameterization schemes: Implications for processes controlling a hairpin turn in the track. $J$. Meteor. Soc. Japan, 97, 577-595.

Scheck, L., S. C. Jones, and V. Heuveline, 2014: Singular vectors for barotropic, hurricane-like vortices in horizontal shear: Structure and perturbation growth mechanisms. J. Atmos. Sci., 71, 1002-1020.

Srinivas, C. V., G. M. Mohan, C. V. Naidu, R. Baskaran, and B. Venkatraman, 2016: Impact of air-sea coupling on the simulation of tropical cyclones in the North Indian Ocean using a simple 3-D ocean model coupled to ARW. J. Geophys. Res., 121, 9400-9421.

Sun, Y., Z. Zhong, L. Yi, T. Li, M. Chen, H. Wan, Y. Wang, and K. Zhong, 2015: Dependence of the relationship between the tropical cyclone track and western Pacific subtropical high intensity on initial storm size: A nu- merical investigation. J. Geophys. Res., 120, 1145111467.

Sun, Y., Z. Zhong, T. Li, L. Yi, S. J. Camargo, Y. Hu, K. Liu, H. Chen, Q. Liao, and J. Shi, 2017: Impact of ocean warming on tropical cyclone track over the western North Pacific: A numerical investigation based on two case studies. J. Geophys. Res., 122, 8617-8630.

Tang, C. K., and J. C. L. Chan, 2014: Idealized simulation of the effect of Taiwan and Philippines topographies on tropical cyclone tracks. Quart. J. Roy. Meteor. Soc., 140, 1578-1589.

Torn, R. D., T. J. Elless, P. P. Papin, and C. A. Davis, 2018: Tropical cyclone track sensitivity in deformation steering flow. Mon. Wea. Rev., 146, 3183-3201.

Velden, C. S., and L. M. Leslie, 1991: The basic relationship between tropical cyclone intensity and the depth of the environmental steering layer in the Australian region. Wea. Forecasting, 6, 244-253.

Wei, N., Y. Li, D.-Z. Zhang, Z. Mai, and S.-Q. Yang, 2016: A statistical analysis of the relationship between uppertropospheric cold low and tropical cyclone track and intensity changes over the western North Pacific. Mon. Wea. Rev., 144, 1805-1822.

Winterbottom, H. R., E. W. Uhlhorn, and E. P. Chassignet, 2012: A design and an application of a regional coupled atmosphere-ocean model for tropical cyclone prediction. J. Adv. Model. Earth Syst., 4, M10002.

Wood, V. T., and L. W. White, 2011: A new parametric model of vortex tangential-wind profiles: Development, testing, and verification. J. Atmos. Sci., 68, 9901006.

Wu, C.-C., T.-H. Li, and Y.-H. Huang, 2015: Influence of mesoscale topography on tropical cyclone tracks: Further examination of the channeling effect. J. Atmos. Sci., 72, 3032-3050.

Wu, L., and X. Chen, 2016: Revisiting the steering principle of tropical cyclone motion in a numerical experiment. Atmos. Chem. Phys., 16, 14925-14936.

Yamaguchi, M., J. Ishida, H. Sato, and M. Nakagawa, 2017: WGNE intercomparison of tropical cyclone forecasts by operational global models: A quarter-century and beyond. Bull. Amer. Meteor. Soc., 98, 2337-2349.

Yamada, H., T. Nasuno, W. Yanase, and M. Satoh, 2016: Role of the vertical structure of a simulated tropical cyclone in its motion: A case study of typhoon Fengshen (2008). SOLA, 12, 203-208.

Zhu, H., U. Wolfgang, and R. K. Smith, 2004: Ocean effects on tropical cyclone intensification and inner-core asymmetries. J. Atmos. Sci., 61, 1245-1258. 